\title{
Resistance to cycloxaprid in Laodelphax striatellus is associated with altered expression of nicotinic acetylcholine receptor subunits
}

Yueliang Zhang ${ }^{\text {a\#}}$, Yangchun Han ${ }^{\text {a\# }}$, Qiong Yang ${ }^{\mathrm{a}}$, Lihua Wang ${ }^{\mathrm{a}}$, Peng $\mathrm{He}^{\mathrm{b}}$, Zewen $\mathrm{Liu}^{\mathrm{c}}$, Huifang Guo ${ }^{\mathrm{a} *}$ and Jichao Fang ${ }^{\mathrm{a} *}$

${ }^{a}$ Institute of Plant Protection, Jiangsu Academy of Agricultural Sciences, Key Lab of Food Quality and Safety of Jiangsu Province-State Key Laboratory Breeding Base, Nanjing 210014, China.

${ }^{\mathrm{b}}$ State Key Laboratory Breeding Base of Green Pesticide and Agricultural Bioengineering, Key Laboratory of Green Pesticide and Agricultural Bioengineering, Ministry of Education, Guizhou University, Guiyang 550025, China.

${ }^{c}$ Key Laboratory of Monitoring and Management of Plant Disease and Insects, Ministry of Agriculture, Nanjing Agricultural University, Nanjing 210095, China.

${ }^{\#}$ Contribute equally

*Corresponding Author:

Jichao Fang and Huifang Guo, plant Protection Research Institute, No. 50, Zhongling Street, Xiaolingwei, Nanjing 210014, Jiangsu Province.

Phone/Fax: +86 2584390891

Email: fangjc@jaas.ac.cn and guohfjaas@163.com

This is the author manuscript accepted for publication and has undergone full peer review but has not been through the copyediting, typesetting, pagination and proofreading process, which may lead to differences between this version and the Version of Record. Please cite this article as doi: $10.1002 / \mathrm{ps} .4757$ 


\section{Abstract}

BACKGROUND: Cycloxaprid is a new oxabridged cis-configuration neonicotinoid insecticide, the resistance development potential and underlying resistance mechanism of which were investigated in the small brown planthopper, Laodelphax striatellus (Fallén), an important agricultural pest of rice.

RESULTS: A cycloxaprid-resistant strain (YN-CPD) only achieved 10 -fold resistance, in contrast to 106-fold resistance to buprofezin and 332-fold resistance to chlorpyrifos achieved after exposure to similar selection pressure, and the cycloxaprid selected line showed no cross-resistance to the buprofezin and chlorpyrifos-selected resistance strains. Moreover, we identified 10 nicotinic acetylcholine receptor (nAChR) subunits from the transcriptome of L. striatellus, and 6 segments had open reading frames (ORFs). While we did not find mutations in the nAChR genes of $L$. striatellus, subunits $L s \pm 1$ and $L s^{2} 1$ exhibited respectively 9.60-fold and 3.36-fold higher expression in the resistant strain, while $L s \pm 8$ exhibited 0.44-fold lower expression. Suppression of $L s \pm l$ through ingestion of $d s L s \pm l$ led to an increase in susceptibility to cycloxaprid.

CONCLUSION: The findings indicate that resistance to cycloxaprid develops slowly compared to other chemicals and without cross-resistance to chlorpyrifos or buprofezin; over-expressed $L s \pm l$ is associated with low cycloxaprid resistance levels, but the importance of over-expressed $L s^{2} 1$ and reduced expression of $L s \pm 8$ could not be excluded. 


\section{INTRODUCTION}

Neonicotinoid insecticides, such as imidacloprid, clothanidin and thiametoxam, are the most commonly used chemical insecticides for pest control and animal health applications. ${ }^{1-3}$ These chemicals have low mammalian toxicity, broad insecticidal spectra and good systemic properties. ${ }^{4}$ Although new neonicotinoid compounds are being generated, there is danger of resistance developing to these chemicals, as has been the case for other pesticides such as organophosphates and pyrethroids. ${ }^{5}$ This is particularly critical because of increasing incidence of resistance to these types of insecticides and cross-resistance, which is now being observed in field pests. ${ }^{6}$

One of the newest neonicotinoids is cycloxaprid, an oxabridged cis-configuration neonicotinoid insecticide that was first developed in 2008 . $^{7}$ If target-site resistance to this new compound evolves, it is likely to target the nicotinic acetylcholine receptors (nAChRs), a superfamily of ligand-gated ion channels that are made up of five subunits. ${ }^{8}$ This pesticide is particularly important for controlling sucking and biting insects, especially imidacloprid-resistant populations of the aphid Aphis gossypii and the brown planthopper Nilaparvata lugens. ${ }^{9-10}$ To understand the long-term viability of this new pesticide, it is important to understand the potential underlying resistance mechanisms and potential for resistance development against cycloxaprid.

Laodelphax striatellus (Fallén) (Homoptera: Delphacidae) is one of the most important pests of rice. It has a widespread distribution in China, 
where it causes serious damage to rice due to the transmission of rice virus diseases or due to the direct effects of sap-sucking from rice stems. ${ }^{11}$ Several management practices had been developed to suppress $L$. striatellus, but its control relies almost exclusively on the application of insecticides, ${ }^{5}$ and cycloxaprid has insecticidal activity against this pest. The first aim of this paper was to compare the potential for cycloxaprid resistance to develop and contrast the rate of resistance development to that for chlorpyrifos and buprofezin based on selection responses in a susceptible (YN) strain. The second aim was to establish patterns of cross-resistance of cycloxaprid to chlorpyrifos and buprofezin. Finally, we considered potential mechanisms responsible for resistance; the nAChRs of $L$. striatellus were validated through transcriptome-based expression and RNA interference technique (RNAi) analysis.

\section{MATERIALS AND METHODS}

\subsection{Insects}

A susceptible (YN) strain of L. striatellus that contained thousands of individuals was collected from Yunnan, China in July 2001 and has been reared without insecticides since that time. The YN-CPF, YN-BPF and YN-CPD strains were derived from the YN strain in the laboratory by discontinuous selection with chlorpyrifos, buprofezin and cycloxaprid for 102 generations, respectively (among 102 generations, 93, 92 and 90 generations exposed to cycloxaprid, chlorpyrifos and buprofezin, respectively). In each generation, approximately 2000 3rd-instar nymphs were separately selected by the rice seedling dip method with average 
mortality of approximately $70 \%{ }^{12}$ The insects were all reared on rice seedlings at $27 \pm 1^{\circ} \mathrm{C}$ under a $14: 10 \mathrm{~h}$ light: dark cycle.

\subsection{Bioassays}

The degree of resistance of the YN, YN-CPF, YN-BPF and YN-CPD strains against chlorpyrifos, buprofezin and cycloxaprid was assayed by the rice seedling dip bioassay method based on previous approaches with a few modifications. ${ }^{12}$ The formulated insecticides were diluted in distilled water to generate six serial dilutions, and every serial dilution contained 1\%o Triton x-100. A group of four rice seedlings was immersed individually into an insecticide dilution for $20 \mathrm{~s}$. After air-drying, the seedlings were placed in disposable plastic cups with moistened paper to maintain the wetness of the rice seedling roots. Fifteen 3rd-instar nymphs were placed in each treated plastic cup. Rice seedlings dipped in distilled water that contained $1 \%$ Triton $\mathrm{x}-100$ were used as a control. For each dilution, three replicates each containing 15 individuals were treated. All tests were maintained at $27( \pm 1)^{\circ} \mathrm{C}$, and mortality was recorded after $48 \mathrm{~h}$, $120 \mathrm{~h}$ and $120 \mathrm{~h}$ for chlorpyrifos, buprofezin and cycloxaprid, respectively. To analyse the synergistic effect of enzyme inhibitors with cycloxaprid, $15 \mathrm{mg} \mathrm{L}^{-1}$ triphenyl phosphate (PBO) was added to each dilution. Bioassay data were subjected to probitanalysis using POLOPLUS software.

\subsection{Identification of $\mathrm{nAChRs}$ in the $L$. striatellus transcriptome}

Total RNA was extracted using the SV Total RNA Isolation system (Promega) according to the manufacturer's protocol. To obtain complete 
transcriptome information, a sample containing RNA pooled from different developmental stages of the $\mathrm{YN}$ individuals was used for transcriptome analysis. The complete transcriptome of $L$. striatellus was created using short-read sequencing technology (Illumina) as previously described. ${ }^{13}$ All gene functions were annotated based on the following databases: $\mathrm{Nr}$ (NCBI non-redundant protein sequences), $\mathrm{Nt}$ (NCBI non-redundant nucleotide sequences), Pfam (Protein family), KOG/COG (Clusters of orthologous groups of proteins), Swiss-Prot (A manually annotated and reviewed protein sequence database), KO (KEGG orthologue database) and GO (gene ontology). We identified nAChRs and nAChR-like genes from the above annotation analysis of the L. striatellus transcriptome. To resolve the homozygosity problems of nAChRs in the transcriptome database, we resequenced these nAChRs by PCR amplification. After candidate nAChRs were obtained, BlastP was performed in NCBI to further support annotation predictions Table S1.

\subsection{Screening of $L$. striatellus nAChRs associated with cycloxaprid resistance}

Total RNA was extracted from 3rd-instar nymphs of the YN and YN-CPD strains using the SV Total RNA Isolation system (Promega). Three independent RNA preparations were made for the YN and YN-CPD strains; each RNA preparation contained 15 individuals. The first-strand cDNA was synthesized from $2 \mu \mathrm{g}$ of total RNA using an oligo(dT)15 primer and Superscript III reverse transcriptase (Promega). The relative expression level of the $10 \mathrm{nAChRs}$ in the YN and YN-CPD strains was determined by qPCR using ADP ribosylation factor (ARF) as a reference 
gene (GenBank number:JF728807). ${ }^{14}$ The primer sequences and the expected size of each PCR product are shown in Table S2. The qPCR was performed on three biological samples for each L. striatellus strain, and each sample was analysed in three technical replicates on an Applied Biosystems 7500 thermocycler. The $20 \mu \mathrm{L}$ PCR mixture contained $10 \mu \mathrm{L}$ of SYBR Premix Ex Taq ${ }^{\mathrm{TM}}$ (Takara, Japan), $1 \mu \mathrm{L}$ of cDNA, $0.4 \mu \mathrm{L}$ of ROX Reference Dye $(50 \times)$, and $0.4 \mu \mathrm{L}$ of $10 \mu \mathrm{M}$ sense and antisense primers in a total volume of $20 \mu \mathrm{L}$. The optimized cycling programme was 1 cycle of $95^{\circ} \mathrm{C}$ for $30 \mathrm{~s}, 40$ cycles of $95^{\circ} \mathrm{C}$ for $5 \mathrm{~s}$, and $60^{\circ} \mathrm{C}$ for $31 \mathrm{~s}$, and a final disassociation stage that was automatically added by the 7500 System SDS software. The relative expression was calculated using the $2^{-" \text { "' } \mathrm{CT}}$ method. ${ }^{15}$ Student's t-test was performed to determine whether the differences in relative expression of the $10 \mathrm{nAChRs}$ in the $\mathrm{YN}$ and YN-CPD strains were significant.

\subsection{Resistance functional analysis through RNA interference technique (RNAi)}

A $403 \mathrm{bp}$ segment of the Lsal cDNA and a $426 \mathrm{bp}$ fragment of the green fluorescent protein $(G F P)$ gene, as a control, were amplified by PCR. Both PCR products were individually subcloned into the pGEM-T easy vector (Promega), and the diluted plasmids were used as templates for the amplification of these target sequences with specific primers that were combined with the T7 RNA polymerase promoter (Table S3). The PCR products were purified with Wizard ${ }^{\circledR}$ SV Gel (Promega) and used as templates for dsRNA synthesis with the T7 Ribomax TM Express RNAi System according to the manufacturer's instructions (Promega). The 
dsRNAs of Lsal and GFP were dissolved in ultrapure water, and the quality and concentrations of the dsRNAs were determined by agarose gel electrophoresis and a Nanodrop 2000 spectrophotometer (Thermo Scientific, Wilmington, DE, USA). Forty 2nd-instar nymphs were carefully transferred into the chamber of a glass cylinder $(12 \mathrm{~cm}$ in length and $2.8 \mathrm{~cm}$ in internal diameter) with a liquid artificial diet between two layers of stretched Parafilm M (Pechiney Plastic Pack ageing Company, Chicago, IL, USA); the stretched Parafilm M was placed at one end of the chamber, and dense breathable gauze was placed at the other end. The diet was changed and dead nymphs removed daily. Six independent preparations were made for $200 \mathrm{mg} / \mathrm{mL}$ ingestion treatment. Forty-five insects were collected at $5 \mathrm{~d}$ after ingestion to determine the expression levels of Lsal using qPCR. A group of insects not fed dsRNA were also tested. Changes in cycloxaprid susceptibility of the YN-CPD strains after RNAi were evaluated using rice seedling dipping methods as mentioned above.

\section{RESULTS}

\subsection{Resistance levels and PBO synergistic assay}

The toxicity of cycloxaprid, chlorpyrifos and buprofezin to the $\mathrm{YN}$, YN-CPD, YN-CPF and YN-BPF strains of L. striatellus as well as cross-resistance to cycloxaprid in the YN-CPF and YN-BPF strains are summarized in Fig. 1 and Table 1. Compared with the YN strain, the YN-CPD, YN-CPF and YN-BPF strains exhibited 10.24-, 106.08- and 331.64-fold resistance to cycloxaprid, chlorpyrifos and buprofezin, 
respectively. These large fold differences occurred despite similar selection pressures and a similar number of selection generations. Synergism experiments showed that there was no significant synergistic effect of PBO on cycloxaprid in both the YN-CPD and YN strains; the relative synergism ratio of $\mathrm{PBO}$ in the cycloxaprid-resistant $\mathrm{YN}-\mathrm{CPD}$ strain was only 1.08 (Table.1).

\subsection{Cross-resistance}

Cross-resistance to cycloxaprid in the YN-CPF and YN-BPF strains was evaluated. The $\mathrm{LC}_{50}$ of cycloxaprid for the YN-CPF and YN-BPF strains of L. striatellus was $5.35 \mathrm{mg} / \mathrm{L}$ and $6.00 \mathrm{mg} / \mathrm{L}$, respectively, as opposed to an $\mathrm{LC}_{50}$ of cycloxaprid for the $\mathrm{YN}$ strain of $5.68 \mathrm{mg} / \mathrm{L}$. These values produce cross-resistance ratios of 0.94 and 1.06, respectively (Table 1). The results showed cycloxaprid had no cross-resistance with chlorpyrifos or buprofezin in the laboratory-selected resistant strains.

\subsection{Molecular cloning of nAChR subunits from $L$. striatellus}

A total of 10 sequences putatively derived from different $n A C h R$ subunits, with high identity (33\% to 99\%) to the nAChRs from other insect species and low E-value $(<5 \mathrm{e}-89)$, were identified from the transcriptome database of L. striatellus. We further validated these sequences through RT-PCR using specific primers (Table S1). Among these, 6 segments had ORFs with amino acid sequences that were similar to previously cloned nAChR subunits found in other insects and feature characteristics of the nAChR gene family. Four putative hydrophobic transmembrane domains and six domains (loops A-F), believed to be important in forming the 
agonist antagonist binding site, are shown in Fig. $2 .^{16-17}$ The Loop F domain is not conserved between nAChR subunits of $N$. lugens and $L$. striatellus. Based on their relative sequence similarity to $\mathrm{AChR}$ subunits from other insects, the putative small planthopper nAChR subunits were named Lsal ( $73 \%$ similarity to al subunit of N. lugens), Lsa8 (95\% similarity to $a 8$ subunit of $N$. lugens), $L^{2} 1$ (99\% similarity to ${ }^{2} 1$ subunit of $N$. lugens), Lsa2 (99\% similarity to a2 variant A of Periplaneta americana), Lsa3 (84\% similarity to a3 variant A of P. americana), Lsa4 (83\% similarity to $a 4$ subunit of $P$. americana), Lsa5 (84\% similarity to a5 of Locusta migratoria), and Lsa6 (84\% similarity to a6 of $P$. americana). The nAChR subunits of L. striatellus are shown in Table S2.

\subsection{Screening the L. striatellus $\mathrm{nAChR}$ subunits associated with cycloxaprid resistance}

Among the 10 nAChRs, $L s \pm 1$ and $L s^{2} 1$ exhibited 9.60-fold and 3.36-fold higher expression levels respectively in the YN-CPD strain when compared to YN strain, while $L s \pm 8$ exhibited a 0.44-fold lower expression level in YN-CPD strain when compared to YN strain (Fig. 3). Furthermore, when the cDNA sequences of nAChRs amplified from the $\mathrm{YN}$ and $\mathrm{YN}-\mathrm{CPD}$ individuals were aligned, no sequence differences were found (Fig. S1).

\subsection{Knockdown of $L s \pm 1$ increases sensitivity to cycloxaprid in the YN-CPD strain}

Because $L s \pm l$ exhibited 9.60-fold higher expression in the YN-CPD strain than the YN strain, we further evaluated the cycloxaprid resistance role of $L s \pm l$ via RNAi. Before conducting the RNAi, candidate siRNA 
target regions in $L s \pm l$ were first calculated through https://www.genscript.com/ssl-bin/app/rnai (Genscript Corporation). Previous studies reported that ingestion of a dose of dsRNA $(0.2 \mathrm{mg} / \mathrm{mL})$ by small rice planthopper nymphs induced highly efficient RNAi for NADPH-cytochrome P450 reductase on the fifth day. ${ }^{18}$ Therefore, 2nd-instar nymphs of $L$. striatellus were fed a dose of $0.2 \mathrm{mg} / \mathrm{mL}$ of $d s L s \pm l$ and $d s G F P$. qPCR data showed that the $L s \pm l$ mRNA levels were dramatically decreased at $5 \mathrm{~d}$, as shown in Fig. 4; ingestion of $0.2 \mathrm{mg} / \mathrm{mL}$ of $d s L s \pm 1$ for $5 \mathrm{~d}$ resulted in 0.65 -fold lower expression than the ingestion of $d s G F P$ and 0.57 -fold lower expression than the ingestion of artificial diet only (control). Then, the $58.18 \mathrm{mg} / \mathrm{L} \mathrm{LC}_{50}$ discriminating dose for the YN-CPD strain was used to investigate differences in susceptibility after RNAi. Knockdown of $L s \pm l$ in the YN-CPD strain significantly increased its susceptibility to cycloxaprid compared with the control. Among these insects, $82.9 \%$ died after cycloxaprid exposure, which was significantly higher mortality than observed in the control group, in which only $57.12 \%$ of the GFP dsRNA-treated and $48.25 \%$ of the native insects died (Fig. 5).

\section{DISCUSSION}

This is the first time that resistance to cycloxaprid has been successfully selected, however, it is clearly difficult to increase resistance, which only reached 10.2-fold for cycloxaprid after 102 generations of selection, compared to 106 and 331-fold resistance for chlorpyrifos and buprofezin, respectively. In addition, these chlorpyrifos and buprofezin resistance strains had no cross-resistance to cycloxaprid, which shows that the mechanisms of resistance conferring protection against chlorpyrifos and 
buprofezin are specific and do not extend their protection to cycloxaprid. In addition, the original strain comes from Chuxiong prefecture of Yunnan province in China and has been reared without insecticides since 2001. This L. striatellus strain had low resistance levels against different insecticides, including chlorpyrifos and buprofezin ${ }^{5}$. Since our study used a laboratory-selected susceptible strain, whether such mechanisms exist in resistant populations in the field deserves further attention.

Cycloxaprid, unlike imidacloprid insecticides, is an oxabridged cis-configuration neonicotinoid insecticide that was first named in China in 2011. ${ }^{19}$ It could serve as a slow-release reservoir for NMI and has selective activity against insect versus mammalian nAChRs. Imidazole (NMI) is a super-potent neonicotinoid, but the photolability of the nitromethylene substituent has restricted its use in agricultural pest control. ${ }^{20-21}$ Previous studies found that binding affinity to the Drosophila nAChR in vitro was approximately 18 -fold higher for NMI than imidacloprid; the Ki values (nM) for displacing $\left[{ }^{3} \mathrm{H}\right]$ imidacloprid binding were 0.12 for NMI and 2.2 for imidacloprid in Drosophila head membranes. ${ }^{22}$ Therefore, a synergistic effect between cycloxaprid and NMI might be responsible for the high potency of cycloxaprid, effectively controlling sucking and biting insects, especially imidacloprid-resistant populations of $N$. lugens and Aphis gossypii. ${ }^{9-10}$

The mode of action of cycloxaprid, similar to imidacloprid, is thought to affect the function of nAChRs. ${ }^{23-24} \mathrm{nAChRs}$ are a type of ligand-gated ion channel that mediate fast synaptic transmission in the insect nervous system and represent targets of neonicotinoid insecticides. Numerous 
nAChR subunits have also been cloned from several insect species, which include N. lugens, Drosophila melanogaster, Anopheles gambiae and Bombyx mori. ${ }^{16,25-27}$ Subunit composition dictates the pharmacological and functional properties of the nAChRs. nAChRs generally exist as five subunits arranged in combination from a family of different subunit subtypes. ${ }^{28}$ We obtained $10 \mathrm{nAChR}$ segments from the transcriptome of $L$. striatellus; among these 6 segments had complete ORFs. Most of the sequences contained conserved regions such as a predicted signal peptide, transmembrane domains and six domains (loops A-F), and all the nAChRs of L. striatellus showed relatively high identity (33\% to 99\%) to the nAChRs from other known species, so we inferred these nAChRs belong to nAChRs of L. striatellus. Moreover, we found the Loop F domain is not conserved between nAChR subunits of $N$. lugens and $L$. striatellus. The reason why this difference occurred deserves further study.

For neonicotinoid insecticides, resistance has been shown to result primarily from detoxification metabolism by $\mathrm{P} 450$ monooxygenases or target-site insensitivity of nAChRs. ${ }^{16,29-30}$ First, we did not find that PBO significantly suppressed the cycloxaprid resistance in the YN-CPD strain. We mainly focused on nAChR target-site resistance in this study, but the presence of additional low-level metabolic mechanisms cannot be discounted. Target insensitivity of nAChRs has been demonstrated in several imidacloprid-resistant insects, such as a point mutation (Y151S) of $N l \pm l$ and $N l \pm 3$ and a mutation (N133D) of $N l^{2} l$ in $N$. lugens that result in imidacloprid insensitivity, and a ${ }^{2}$ subunit mutation (R81T) in $A$. 
gossypii that is associated with both a laboratory-selected population and an imidacloprid-resistant field population. ${ }^{16,31-32}$ Except for the target mutations, few reports have described quantitative changes of nAChRs that could also be involved in neonicotinoid insecticide resistance, the clearest evidence is that a decrease in abundance of the Nla 8 subunit conferred resistance to imidacloprid in $N$. lugens ${ }^{33}$ investigations have also found a reduction in \pm 2 expression in $M$. domestica associated with field neonicotinoid resistance; moreover, \pm 10 and ${ }^{2} 1$ of Acyrthosiphon pisum appear to increase expression but ${ }^{2} 2$ of $A$. pisum showed decreased expression when treated with neonicotinoid resistance. ${ }^{34-35}$ In this study, we did not find mutations in $\mathrm{nAChR}$ genes separating the $\mathrm{YN}$ and YN-CPD individuals, and expression analysis found different expression levels of $L s \pm 1, L s^{2} 1$ and $L s \pm 8$, especially 9.60-fold higher expression of $L s \pm l$ associated with low level cycloxaprid resistance. However, the sequence alignment needs to be further investigated following the cycloxaprid resistance increase (when the resistance folds developed above 100-fold) because target mutations are the main resistance mechanism for neonicotinoid insecticides ${ }^{16,31-32}$.

Previous studies had reported that the ingestion of double-stranded RNA (dsRNA) could effectively suppress target genes in small rice planthoppers. ${ }^{18,36-37}$ In this study, we considered expression of $L s \pm l$ to evaluate its cycloxaprid resistance function in the YN-CPD strain. Ingestion of $0.2 \mathrm{mg} / \mathrm{mL} d s L s \pm l$ for $5 \mathrm{~d}$ resulted in the effective suppression of $L s \pm l$ expression, and it significantly increased susceptibility to cycloxaprid compared with the control, further 
confirming the involvement of $L s \pm l$ overexpression in cycloxaprid resistance. Although cycloxaprid and imidacloprid have common targets, they might have different modes of action, over-expressed $L s \pm l$ conferring cycloxaprid resistance was different from decreasing amount of Nla 8 reducing imidacloprid resistance potency; over-expressed $L s \pm l$ provides more competitive combination reserves for both cycloxaprid and acetylcholine, so the overproduction nAChRs could extricate oneself involve in normal physiological function when confronted with insecticide pressure. Moreover, it did not significantly suppress the expression of $L s^{2} 1$ or $L s a 8$, and the mortality rate of L. striatellus is high after $d s L s^{2} 1$ or $d s L s a 8$ ingestion (data not shown), but the importance of over-expressed $L s^{2} 1$ and reduced expression of $L s \pm 8$ in cycloxaprid resistance could not be excluded. A previous study found CRISPR-CAS based gene editing could successfully knock down Drosophila nAChR expression $^{38}$, so this new technology needs to be explored further in $L$. striatellus to verify nAChR resistance function.

In the present study, although our findings provide reliable evidence that cycloxaprid resistance is hard to obtain, and low cycloxaprid resistance of L. striatellus is associated with altered expression of $\mathrm{nAChR}$ subunits, some puzzling questions should be answered in subsequent study. 1) The correlation between overexpressed $L s \pm l$ and fitness cost situations in YN-CPD strain deserves further research; 2) the mRNA levels of nAChR subunits do not always reveal the protein levels, protein quantification analysis needs to be conduct to confirm these; 3) Cycloxaprid radio-ligand binding assays before and after RNAi of $L s \pm l$ 
in YN-CPD individuals should be conducted.

\section{ACKNOWLEDGEMENTS}

The authors sincerely thank Prof. Ary Hoffmann for proofreading and editing the manuscript. This work was supported by National Natural Science Foundation of China (31301697), the Jiangsu Fund for Innovation of Agricultural Science and Technology (CX(15)1055), the foundation of Jiangsu Province Academy of Agricultural Science (6111609), the food Production Engineering of China (2016YFD0300706).

\section{REFERENCES}

1 Koo H N, An J J, Park S E, Kim J I and Kim G H, Regional susceptibilities to 12 insecticides of melon and cotton aphid, Aphis gossypii (Hemiptera: Aphididae) and a point mutation associated with imidacloprid resistance. Crop Prot 55: $91-97$ (2014).

2 Kayser H, Lee C, Decock A, Baur M, Haettenschwiler J and Maienfisch P, Comparative analysis of neonicotinoid binding to insect membranes: I. A structure-activity study of the mode of H-3 imidacloprid displacement in Myzus persicae and Aphis craccivora. Pest Manag Sci 60: 945-958 (2004).

3 Jeschke $\mathrm{P}$ and Nauen $\mathrm{R}$, Neonicotinoids-from zero to hero in insecticide chemistry. Pest Manag Sci 64: 1084-1098 (2008).

4 Tomizawa $\mathrm{M}$ and Casida $\mathrm{J}$ E, Selective toxicity of neonicotinoids attributable to specificity of insect and mammalian nicotinic receptors. Annu Rev Entomol 48: 339-364 (2003). 
5 Wang L H, Fang J C and Liu B S, Relative toxicity of insecticides to Laodelphax striatellus (Fallén) (Homoptera: Delphacidae) and resistance of the field populations from different areas of east China (in Chinese with English summary). Acta Entomol Sin 51: 930-937 (2008).

$6 \mathrm{Xu} \mathrm{L}, \mathrm{Wu} M$ and Han $\mathrm{Z}$ J, Biochemical and molecular characterisation and cross-resistance in field and laboratory chlorpyrifos-resistant strains of Laodelphax striatellus (Hemiptera: Delphacidae) from eastern China. Pest Manag Sci 70:1118-1129 (2014).

7 Shao X S, Zhang W W, Peng Y Q, Li Z, Tian Z Z and Qian X H, cis-Nitromethylene neonicotinoids as new nicotinic family: Synthesis, structural diversity, and insecticidal evaluation of hexahydroimidazo 1,2-alpha pyridine. Bioorg Med Chem Lett 18: 6513-6516 (2008).

8 Lester H A, Dibas M I, Dahan D S, Leite J F and Dougherty D A, Cys-loop receptors: new twists and turns. Trends Neurosci 27: 329-336 (2004).

9 Cui L, Qi H L, Yang D B, Yuan H Z, Rui C H, Cycloxaprid: A novel cis-nitromethylene neonicotinoid insecticide to control imidacloprid-resistant cotton aphid (Aphis gossypii). Pestic Biochem Physiol 132: 96-101 (2016).

10 Shao X S, Fu H, Xu X Y, Xu X L, Liu Z W, Li Z and Qian X H, Divalent and Oxabridged Neonicotinoids Constructed by Dialdehydes and Nitromethylene Analogues of Imidacloprid: Design, Synthesis, Crystal Structure, and Insecticidal Activities. J Agr Food Chem 58: 2696-2702 (2010).

11 Kisimoto R, Genetic variation in the ability of a planthopper vector; 
Laodelphax striatellus (Fallén) to acquire the rice stripe virus. Virology 32:144-152 (1967).

12 Wang $\mathrm{L} \mathrm{H}$, Zhang $\mathrm{Y} \mathrm{L}$, Han $\mathrm{Z} \mathrm{J}$, Liu $\mathrm{Y} \mathrm{H}$ and Fang $\mathrm{J} \mathrm{C}$, Cross-resistance and possible mechanisms of chlorpyrifos resistance in Laodelphax striatellus (Fallen). Pest Manag Sci 66:1096-1100 (2010).

13 Xue J A, Bao Y Y, Li B L, Cheng Y B, Peng Z Y etal., Transcriptome Analysis of the Brown Planthopper Nilaparvata lugens. Plos One 5: e14233 (2010).

14 Zhang Y L, Guo H F, Yang Q, Li S, Wang L H, Zhang G F and Fang J $\mathrm{C}$, Overexpression of a $\mathrm{P} 450$ gene $(C Y P 6 C W 1)$ in buprofezin-resistant Laodelphax striatellus (Fallen). Pestic Biochem Physiol 104: 277-282 (2012).

15 Livak K J and Schmittgen T D, Analysis of relative gene expression data using real-time quantitative PCR and the 2(T)(-Delta Delta C) method. Methods 25: 402-408 (2001).

16 Liu Z, Wartin M S, Lansdell S J et al, A nicotinic acetylcholine receptor mutation conferring target-site resistance to imidacloprid in Nilaparvata lugens (brown planthopper). PANS 102: 8420-8425 (2005).

17 Thany P S H, Insect Nicotinic Acetylcholine Receptors. Adv Exp Med Biol 683:1-117 (2011).

18 Zhang Y L, Wang Y M, Wang L H, Yao J, Guo H F and Fang J C, Knockdown of NADPH-cytochrome P450 reductase results in reduced resistance to buprofezin in the small brown planthopper, Laodelphax striatellus (fallén). Pestic Biochem Physiol 127: 21-27 (2016).

19 Li C, Xu X Y, Li J Y, Ye Q F and Li Z, Radiosynthesis of 
tritium-labeled novel nitromethylene neonicotinoids compounds with (NaBH4)-H-3. J Labelled Compd Rad 54: 256-259 (2011).

20 Izuru Yamamoto J E C, Nicotinoid Insecticides and the Nicotinic Acetylcholine Receptor. Springer: New York 978-4-431-68011-6 (1999).

21 Kleier D, Holden I, Casida J E and Ruzo L O, Novel photoreactions of an insecticidal nitromethylene heterocycle. J Agr Food Chem 33: 998-1000 (1985).

22 Zhang N J, Tomizawa M and Casida J E, Structural features of azidopyridinyl neonicotinoid probes conferring high affinity and selectivity for mammalian alpha 4 beta 2 and Drosophila nicotinic receptors. J Med Chem 45: 2832-2840 (2002).

23 Ohno I, Tomizawa M, Durkin K A, Naruse Y, Casida J E and Kagabu S, Molecular Features of Neonicotinoid Pharmacophore Variants Interacting with the Insect Nicotinic Receptor. Chem Res Toxicol 22: 476-482 (2009).

24 Tomizawa M, Maltby D, Talley T T, Durkin K A, Medzihradszky K F, Burlingame A L, Taylor P and Casida J E, Atypical nicotinic agonist bound conformations conferring subtype selectivity. PNAS 105: 1728-1732 (2008).

25 Sattelle D B, Jones A K, Sattelle B M, Matsuda K, Reenan R and Biggin P C, Edit, cut and paste in the nicotinic acetylcholine receptor gene family of Drosophila melanogaster. Bioessays 27: 366-376 (2005).

26 Jones A K, Grauso M and Sattelle D B, The nicotinic acetylcholine receptor gene family of the malaria mosquito, Anopheles gambiae. Genomics 85:176-187 (2005). 
27 Shao Y M, Dong K and Zhang C X, The nicotinic acetylcholine receptor gene family of the silkworm, Bombyx mori. Bmc Genomics $8: 324$ (2007).

28 Karlin A, Emerging structure of the nicotinic acetylcholine receptors. Nat Rev Neurosci 3: 102-114 (2002).

29 Zhang Y X, Yang Y X, Sun H H and Liu Z W, Metabolic imidacloprid resistance in the brown planthopper, Nilaparvata lugens, relies on multiple P450 enzymes. Insect Biochem Mol Biol 79: 50-56 (2016).

30 Zhang J H, Zhang Y X, Wang Y C, Yang Y X, Cang X Z and Liu Z W, Expression induction of $\mathrm{P} 450$ genes by imidacloprid in Nilaparvata lugens: A genome-scale analysis. Pestic Biochem Physiol 132: 59-64 (2016).

31 Zhang Y X, Liu Z W, Han Z J, Song F, Yao X M, Shao Y and Li J, Millar N S, Functional co-expression of two insect nicotinic receptor subunits (Nl alpha 3 and $\mathrm{Nl}$ alpha 8) reveals the effects of a resistance-associated mutation ( $\mathrm{Nl}$ alpha 3(Y151S)) on neonicotinoid insecticides. J Neurochem 110: 1855-1862 (2009).

32 Hirata K, Kiyota R, Matsuura A, Toda S, Yamamoto A and Iwasa T, Association between the R81T mutation in the nicotinic acetylcholine receptor betal subunit of Aphis gossypii and the differential resistance to acetamiprid and imidacloprid. J Pestic Sci 40: 25-31(2015).

33 Zhang Y X, Wang X, Yang B J, Hu Y Y, Huang L X, Bass C and Liu Z $\mathrm{W}$, Reduction in mRNA and protein expression of a nicotinic acetylcholine receptor 8 subunit is associated with resistance to imidacloprid in the brown planthopper, Nilaparvata lugens. J Neurochem 
135: 686-694 (2015).

34 Markussen M D K and Kristensen M, Low expression of nicotinic acetylcholine receptor subunit $\mathrm{Md}$ alpha 2 in neonicotinoid-resistant strains of Musca domestica L. Pest Manag Sci 66: 1257-1262 (2010).

35 Taillebois E, Beloula A, Quinchard S, Jaubert-Possamai S, Daguin A, Servent D, Tagu D and Thany S H, Tricoire-Leignel H, Neonicotinoid Binding, Toxicity and Expression of Nicotinic Acetylcholine Receptor Subunits in the Aphid Acyrthosiphon pisum. Plos One 9: e96669 (2014). 36 Jia S, Wan P J, Zhou L T, Mu L L and Li G Q, Knockdown of a putative Halloween gene Shade reveals its role in ecdysteroidogenesis in the small brown planthopper Laodelphax striatellus. Gene 531: 168-174 (2013).

37 Wan P J, Jia S, Li N, Fan J M and Li G Q, RNA Interference Depletion of the Halloween Gene Disembodied Implies its Potential Application for Management of Planthopper Sogatella furcifera and Laodelphax striatellus. Plos One 9: e86675 (2014).

38 Somers J, Luong $\mathrm{H} \mathrm{N} \mathrm{B}$, Mitchell J, Batterham P and Perry T, Pleiotropic Effects of Loss of the D alpha 1 Subunit in Drosophila melanogaster: Implications for Insecticide Resistance. Genetics 205: 263-271(2017). 


\section{Tables}

Table 1. The level of resistance to cycloxaprid, chlorpyrifos and buprofezin in the YN, YN-CPD, YN-CPF and YN-BPF strains and PBO synergism between YN and YN-CPD strains as well as cross-resistance to cycloxaprid in the YN-CPF and YN-BPF strains of Laodelphax striatellus.

\begin{tabular}{|c|c|c|c|c|c|c|}
\hline Strain & Insecticides & Slope $( \pm$ SE $)$ & $\mathrm{LC}_{50}\left(\mathrm{mg} \mathrm{L}^{-1}\right)(95 \% \mathrm{CL})$ & $\mathrm{RR}^{\mathrm{a}}$ & $\mathrm{CR}^{\mathrm{b}}$ & RSRc \\
\hline YN & Cycloxaprid & $2.39( \pm 0.29)$ & $5.68(4.58-6.90)$ & & & \\
\hline $\mathrm{YN}+\mathrm{PBO}$ & Cycloxaprid & $2.51( \pm 0.32)$ & $5.51(4.43-6.69)$ & & & \\
\hline YN & Chlorpyrifos & $2.04( \pm 0.28)$ & $8.09(6.24-10.9)$ & & & \\
\hline $\mathrm{YN}$ & Buprofezin & $2.23( \pm 0.28)$ & $22.5(18.3-28.0)$ & & & \\
\hline YN-CPF & Cycloxaprid & $2.29( \pm 0.29)$ & $5.35(4.26-6.54)$ & & 0.94 & \\
\hline YN-CPF & Chlorpyrifos & $1.59( \pm 0.35)$ & $2683(1916-5027)$ & 331 & & \\
\hline YN- BPF & Cycloxaprid & $2.03( \pm 0.33)$ & $6.00(4.71-7.69)$ & & 1.06 & \\
\hline YN-BPF & Buprofezin & $1.69( \pm 0.34)$ & $2382(1779-3759)$ & 106 & & \\
\hline YN-CPD & Cycloxaprid & $1.64( \pm 0.32)$ & $58.2(43.8-82.1)$ & 10.2 & & \\
\hline$(\mathrm{YN}-\mathrm{CPD})+\mathrm{PBO}$ & Cycloxaprid & $2.10( \pm 0.28)$ & $52.5(35.9-72.9)$ & & & 1.08 \\
\hline
\end{tabular}


a RR (resistance ratio) $=\mathrm{LC}_{50}$ of YN-CPF, YN-BPF or YN-CPD strains $/ \mathrm{LC}_{50}$ of YN strain.

b CR (cross-resistance ratio) $=\mathrm{LC}_{50}$ of $\mathrm{YN}-\mathrm{CPF}$ or $\mathrm{YN}-\mathrm{BPF}$ strains/LC $\mathrm{C}_{50}$ of $\mathrm{YN}$ strain for cycloxaprid.

${ }^{\mathrm{c}}$ RSR (relative synergism ratio) $=$ synergism ratio of YN-CPD strain/synergism ratio of YN strain. 
Figure 1. Fold change in resistance of L. striatellus exposed to chlorpyrifos, buprofezin or cycloxaprid when compared to an unselected control strain across 102 culture generations. The resistance fold was calculated as $\mathrm{LC}_{50}$ of $\mathrm{YN}-\mathrm{CPF}$, YN-BPF or YN-CPD strain/LC $\mathrm{C}_{50}$ of $\mathrm{YN}$ strain.

Figure 2. Amino acid sequence alignment of $L$. striatellus $\mathrm{nAChR}$ subunits. Also shown are the sequences of the Nilaparvata lugens subunits. Predicted signal peptide (SP) and transmembrane domains (M1-M4) are underlined; the locations of six domains (loops A-F) believed to be important for forming the agonist/antagonist binding site are also indicated.

Figure 3. Fold change in expression level of 10 L. striatellus nAChRs in the YN-CPD strain compared with the YN strain determined by qPCR. Data are presented as the average of three biological replicates, each conducted in duplicate and normalized to a control gene with error bars representing SEM. The change in expression level was calculated using the $2^{-", "} \mathrm{CT}$ method. Asterisks indicate significant differences $(* *$ for $\mathrm{P}<$ $0.01, *$ for $\mathrm{P}<0.05$ ) by Student's t-test.

Figure 4. $L s \pm 1$ mRNA expression levels in native, nymphs that ingested artificial feed; $d s G F P$, nymphs that ingested dsRNA of green fluorescent protein $(G F P)$; and $d s \pm 1$, nymphs that ingested dsRNA of $L s \pm l$. The time from ingestion was $5 \mathrm{~d}$, and the dose was $200 \mu \mathrm{g} / \mathrm{mL}$. The levels of $L s \pm l$ transcription in $L s \pm l$ dsRNA-treated and native-treated insects were normalized to that in $d s G F P$-treated individuals. Data are presented as the average of three biological replicates, each conducted in duplicate and normalized to a control gene with error bars representing SEM. Means depicted with different letters are significantly different $(\mathrm{P}<0.05$, t-test $)$.

Figure 5. Mortality of $L s \pm l$-silenced $L$. striatellus nymphs after cycloxaprid treatment. Native (nymphs that ingested artificial feed), $d s G F P$ (nymphs that ingested dsRNA GFP), and $d s L s \pm l$ (nymphs that 
ingested dsRNA $L s \pm l$ ). Data are presented as the average of three biological replicates, each conducted in duplicate and normalized to a control gene with error bars representing SEM. Means depicted with different letters are significantly different $(\mathrm{P}<0.05$, t-test $)$.

This article is protected by copyright. All rights reserved. 


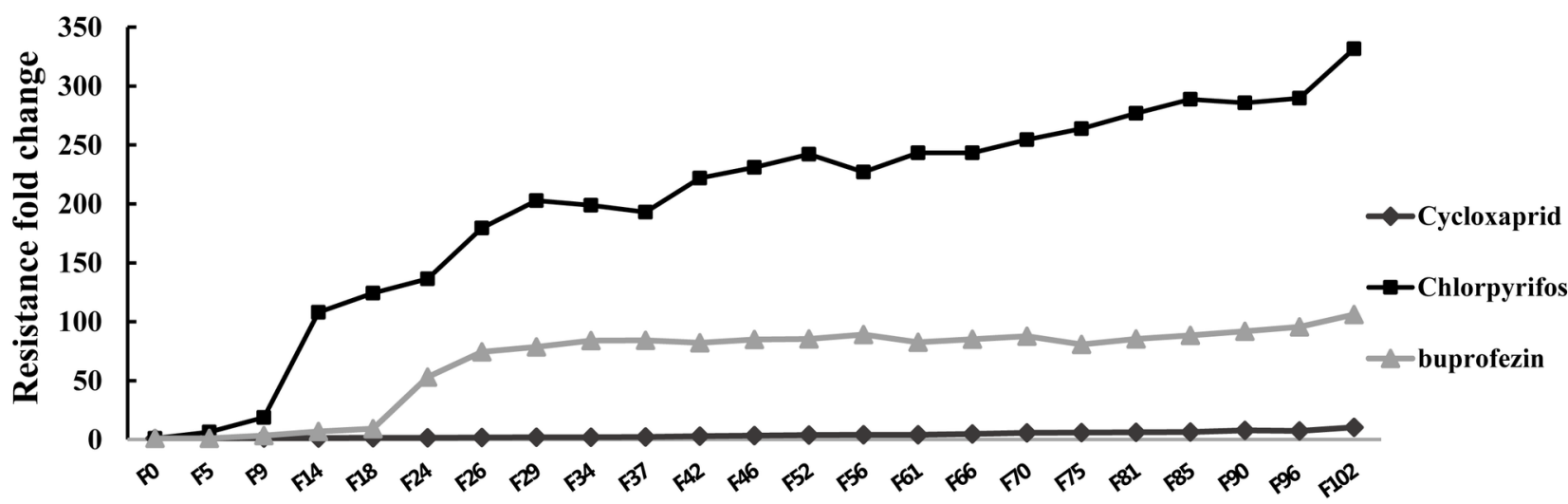

Generation

PS_4757_Fig1.tif

This article is protected by copyright. All rights reserved. 


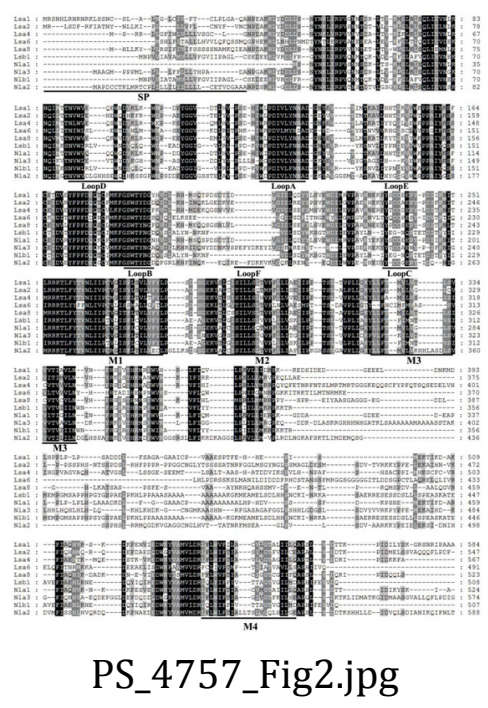

This article is protected by copyright. All rights reserved. 


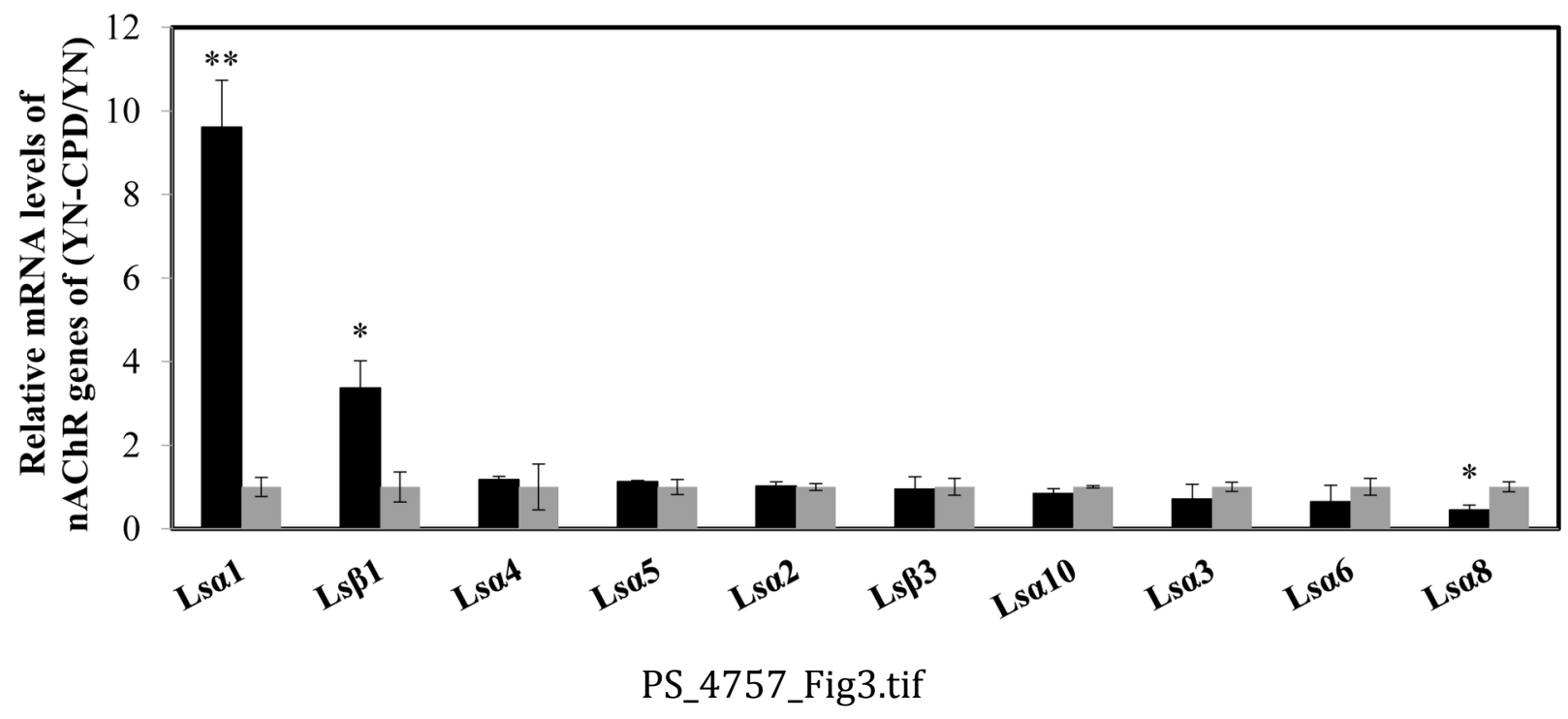

This article is protected by copyright. All rights reserved. 


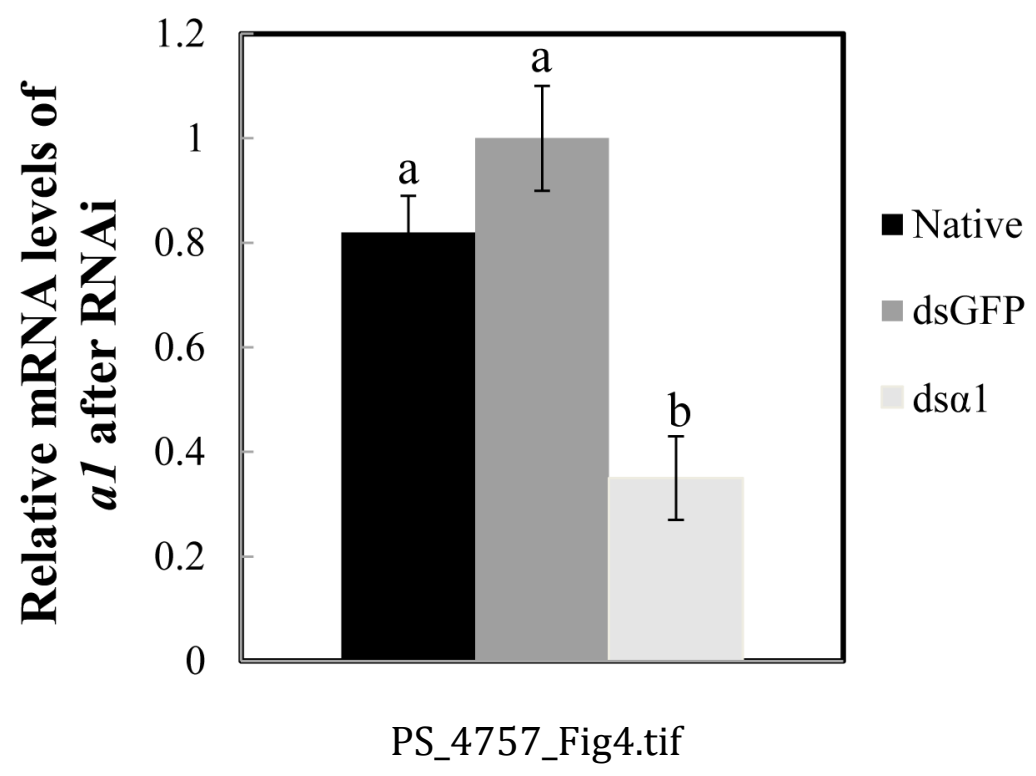

This article is protected by copyright. All rights reserved. 


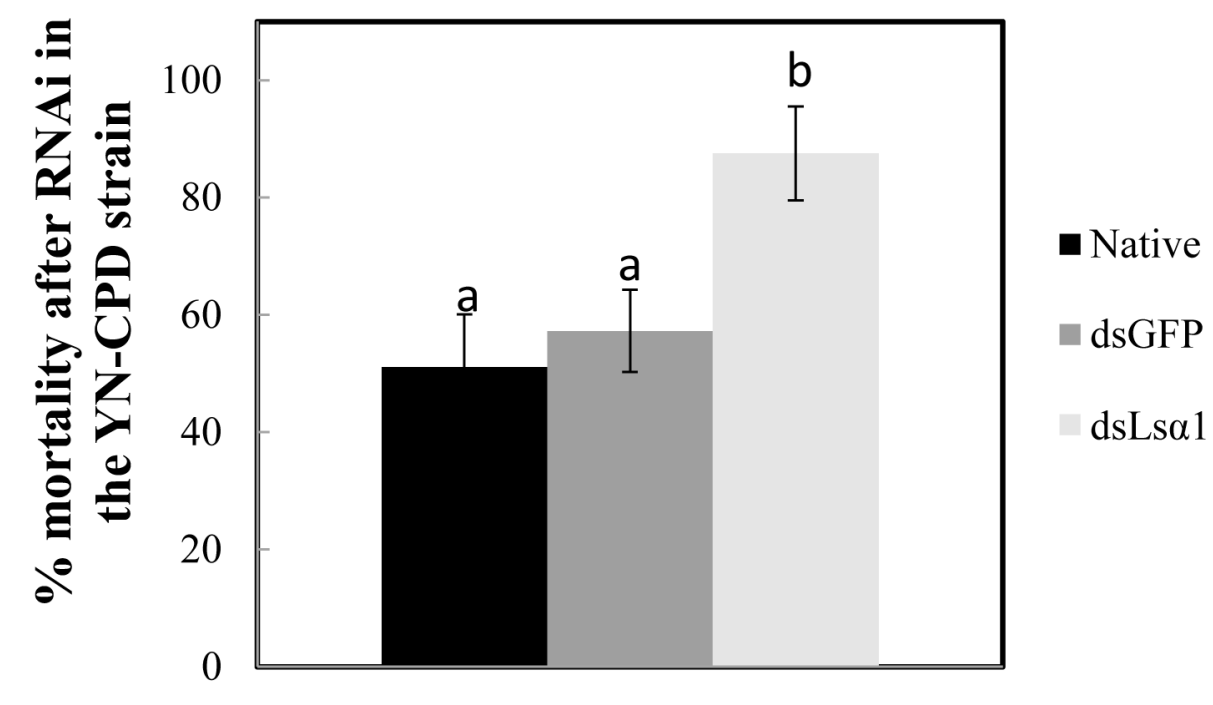

PS_4757_Fig5.tif 


\section{University Library}

\section{- M M N E R VA A gateway to Melbourne's research publications}

Minerva Access is the Institutional Repository of The University of Melbourne

Author/s:

Zhang, Y;Han, Y;Yang, Q;Wang, L;He, P;Liu, Z;Li, Z;Guo, H;Fang, J

Title:

Resistance to cycloxaprid in Laodelphax striatellus is associated with altered expression of nicotinic acetylcholine receptor subunits.

Date:

2018-04

Citation:

Zhang, Y., Han, Y., Yang, Q., Wang, L., He, P., Liu, Z., Li, Z., Guo, H. \& Fang, J. (2018).

Resistance to cycloxaprid in Laodelphax striatellus is associated with altered expression of nicotinic acetylcholine receptor subunits.. Pest Management Science, 74 (4), pp.837-843. https://doi.org/10.1002/ps.4757.

Persistent Link:

http://hdl.handle.net/11343/294034 